Personality, psychopathology and health through the lens of interpersonal relatedness and self-definition

Patrick Luyten, $\mathrm{PhD}^{\mathrm{a}, \mathrm{b}}$

${ }^{a}$ Faculty of Psychology and Educational Sciences, University of Leuven, Belgium

${ }^{b}$ Research Department of Clinical, Educational and Health Psychology, University College London, UK

In press: Journal of the American Psychoanalytic Association

Contact address:

Patrick Luyten, $\mathrm{PhD}$

University of Leuven

Tiensestraat 102 pobox 3722

3000 Leuven

Belgium

e-mail: patrick.luyten@kuleuven.be 


\begin{abstract}
This paper reviews Sidney J. Blatt's seminal contributions in the domain of personality development, psychopathology, and health, which rank among the most well-researched and empirically supported psychoanalytic theories. Blatt is primarily known for his so-called twopolarities model of personality development, which argues that personality development evolves through a dialectic synergistic interaction between two fundamental developmental psychological processes across the life span-the development of interpersonal relatedness on the one hand, and of self-definition on the other. Within this approach, psychopathology is viewed as an attempt to find a balance, however distorted, between relatedness and selfdefinition. Neurobiological research has confirmed the intrinsic and dialectic relationship between the development of self-definition and relatedness in the development of the neural circuits subserving these capacities, which has also important implications for physical health. Research relevant to each of these assumptions is reviewed, and the influence Blatt's approach has had on reintroducing the importance of psychodynamic factors in contemporary psychology and psychiatry, including in DSM-5, is discussed.
\end{abstract}




\section{Personality, Psychopathology and Health through the Lens of Interpersonal Relatedness and Self-Definition}

Faced with the task of providing a summary of Sidney J. Blatt's extensive and monumental contributions to our understanding of personality development in relation to mental and physical health, spanning over 50 years of research, one can feel only trepidation. Yet, the basic gist of his views on the relationships among normal and disrupted personality development in relation to mental health can be relatively easy summarized and are deceptively simple. Basically, Blatt assumed that personality development evolves through a dialectic synergistic interaction between two fundamental developmental psychological lines across the life span, that is, the development of interpersonal relatedness on the one hand and of self-definition on the other. Various forms of psychopathology are viewed within this approach as attempts to find a balance, however distorted, between issues of relatedness and self-definition, leading to a temporary or chronic excessive, distorted and often defensive emphasis on one or the other of these two developmental lines.

In this paper, I briefly review the core assumptions of Blatt's approach to normal and disrupted personality development and the empirical basis of these assumptions. I also review recently emerging findings demonstrating that the human capacity for interpersonal relatedness and self-definition are closely intertwined at the neurobiological level. I discuss the influence of disruptions of the dialectic interaction between relatedness and self-definition on physical health, which appear to be primarily mediated through impairments in the developing stress system and associated biological systems. 


\section{Blatt's approach to normal and disrupted personality development}

Although hundreds of empirical studies have led to further theoretical refinements of Blatt's formulations, demonstrating the progressive nature of the research program that was initiated by these initial formulations (Lakatos, 1976), the basic assumptions of Blatt's so-called twopolarities model of personality development appear to have withstood the test of time.

Consistent with basic psychoanalytic assumptions, for Blatt there is no neat distinction between "normal" and "abnormal" or disrupted personality development (Blatt, 2008; Blatt and Luyten, 2009; Blatt and Shichmann, 1983). His views also do not assume a neat distinction between normal variations in personality development, "symptom disorders", and “personality disorders.” As I will discuss in more detail later, mainstream psychology and psychiatry have rediscovered the validity of both of these latter assumptions, and Blatt's work in this context has had a profound influence, not least on the most recent formulations in DSM-5 (American Psychiatric Association, 2013; Luyten and Blatt, 2015; Skodol and Bender, 2009).

Briefly, Blatt viewed personality development as proceeding through a continuous dialectic interaction between the capacities for interpersonal relatedness and self-definitionthat is, the capacity to establish and maintain, respectively, (a) reciprocal, meaningful, and personally satisfying interpersonal relationships, and (b) a coherent, realistic, differentiated, and essentially positive sense of self or an identity (see Figure 1). Influenced by Anna Freud's concept of developmental lines (Freud, 1963), Blatt considered these two capacities to be central to understanding both adaptive and disrupted human psychological development, and thus vulnerability to psychopathology.

As reviewed by Beebe and colleagues in this issue, from early infancy onward, experiences of interpersonal relatedness — which include feelings of mutuality and gratifying involvement, but also experiences of incompatibility, misunderstanding and separation—are 
thought to contribute to the development of self-definition, which then in turn is expected to foster the capacity for interpersonal relatedness, and vice versa (Blatt and Behrends, 1987). Hence, human beings are seen as fundamentally interpersonal in nature. In Blatt's view, even our feelings of selfhood have their roots in experiences of mutuality as well as incompatibilities and misunderstandings with others, typically beginning in interactions with attachment figures. Influenced by object relations theory and attachment formulations, Blatt viewed experiences of mutuality and incompatibility as instrumental in the need to develop increasingly complex, differentiated, and integrated representations of self and others that influence and guide beliefs and expectations about oneself, others, relationships, and the world (Blatt, 2008).

From this perspective, both adaptive and disrupted personality are seen as modes of adaptation that result from variations and disruptions in the synergistic interaction between relatedness and self-definition throughout the life span (Luyten and Blatt, 2011). These attempts to find a balance between relatedness and autonomy can be more or less adaptive for the individual and/or his/her environment. According to Blatt (2008), the point of equilibrium that is achieved between relatedness and self-definition depends on a complex set of interactions among psychological, sociological, cultural, historical, and biological factors (see Figure 1). This leads individuals, even within the normal range, to place a somewhat greater emphasis on one or the other of these dimensions. For instance, at least in Western cultures, there would be a tendency for women to place somewhat greater emphasis on relatedness, while men would tend to place somewhat greater emphasis on self-definition. Within collectivistic or interdependent cultures, relatedness would be valued more, while in individualistic or independent cultures, self-definition is considered to be more normative (Kitayama et al., 1997; Markus and Kitayama, 1991; Triandis, 2001). Similarly, as reviewed below, parenting styles have been shown to influence the development of a greater emphasis 
on relatedness versus self-definition.

Blatt and colleagues (Blatt and Shichman, 1983) also used the term anaclitic to refer to individuals whose personality organization was focused predominantly on interpersonal relatedness. This notion was taken from the Greek anaklitos, meaning to rest or lean on, a term that was used to refer to Freud's (1905) description of individuals who have a strong need to enter into relationships that rest on or lean on early experiences with caregivers, the so-called Anlehnungstypus der Objektwahl ("anaclitic object choice"). The use of the notion anaclitic was also inspired by Spitz's (1945) description of a type of "anaclitic depression", marked by feelings of lethargy and hopelessness, in infants who were separated from their primary caregiver(s) and who suffered emotional deprivation. In turn, Blatt and colleagues (Blatt and Shichman, 1983) used the term introjective to refer to individuals whose personality organization primarily evolves around self-definition, building on Freud's (1915) notion that the self is developed through a process of introjection or identification with significant others.

Anaclitic and introjective personality styles are thus thought to reflect normal variations within personality development, each with their particular experiential mode, preferred forms of cognition, defenses, and adaptation, unique qualities of interpersonal relatedness, and specific forms of object representation and self-representation (see Table 1).

Although these personality configurations represent normal variations, in the extreme, they give rise to what consensually is defined as psychopathology. Yet, what is important is that even the different forms of psychopathology that are distinguished within a given culture cannot be considered to be static end-states but represent attempts to maintain a balance, however maladaptive, between relatedness and self-definition. Psychopathology thus reflects dynamic conflict-defense constellations (Luyten and Blatt, 2011; McWilliams, 2011). According to Blatt, these maladaptive attempts at adaptation typically involve an excessive 
emphasis on one developmental line and the neglect of the other (Blatt, 2008), leading to a distinction between anaclitic and introjective psychopathology. Anaclitic psychopathology involves, at different developmental levels, a distorted and rigid, one-sided emphasis on interpersonal relatedness; the development of autonomy and self-definition is defensively neglected and/or avoided. Typical examples of anaclitic types of psychopathology include schizophrenia, histrionic, dependent, and borderline personality disorders, and anaclitic depression. In these disorders, relatedness is emphasized at the cost of the development of autonomy and identity; excessive dependency on others is the common theme. Introjective psychopathology, in contrast, is characterized, at different developmental levels, by a distorted and one-sided emphasis on self-definition; an excessive emphasis on the self and autonomy and the defensive neglect of relatedness is the common theme in these disorders. Examples are paranoid schizophrenia and paranoid, obsessive-compulsive, self-critical depressive, and narcissistic personality disorders.

Finally, the more excessive the distorted and often defensive neglect of the other line, the more the attempt at finding a balance is likely to fail, particularly in the longer term, and the more it is associated with psychological costs for the individual and his/her environment. As I will discuss in more detail below, recent extensions of the model have begun to outline the biological costs associated with disruptions of the normal dialectic between relatedness and self-definition, and their implications for physical health (Luyten and Blatt, 2013).

This so-called "double helix theory of personality development" subsequently led to broad-ranging research, particularly as similarities between Blatt's views and those of other prominent theorists across different theoretical orientations - ranging from philosophy to psychology and psychoanalysis—quickly became apparent (for a review, see Blatt, 2008). Subsequent research has shown theoretical and empirical overlaps with similar theories that propose that interpersonal relatedness and self-definition can be seen as central organizational 
features of personality development. In these theories, the respective dimensions have been variously referred to as surrender and autonomy (Angyal, 1951), communion and agency (Bakan, 1966; Pincus, 2005), affiliation or intimacy and achievement or power (McAdams, 1985; McClelland, 1985), relatedness and autonomy/competence (Deci and Ryan, 2012; Ryan and Deci, 2000), attachment anxiety and attachment avoidance (Mikulincer and Shaver, 2007; Sibley, 2007), and sociotropy and autonomy (Beck, 1983; Clark and Beck, 1999). What these theories have in common is that they all propose that adaptive personality development entails a balance between interpersonal relatedness and self-definition, whereas psychopathology involves a temporary or chronic disruption of this balance (Luyten and Blatt, 2011). Within attachment theory, for instance, adaptive personality is conceptualized as a balance between relatedness and self-definition, as expressed in low levels of attachment anxiety and avoidance (Mikulincer \& Shaver, 2007). Insecure attachment styles, in turn, are thought to represent different types of imbalance between these dimensions. Avoidant attachment, for instance, entails "discomfort with closeness" and "discomfort with depending on others" (Mikulincer \& Shaver, 2007, p. 87), representing an exaggerated emphasis on selfdefinition and a defensive avoidance of interpersonal relatedness. Attachment anxiety is typically characterized by "fear of rejection and abandonment" (Mikulincer \& Shaver, 2007, p. 155), representing an overemphasis on relatedness and a defensive avoidance of feelings of autonomy and self-affirmation (i.e., self-definition).

\section{From the clinic to the lab and back}

Blatt's initial formulations were in part derived from his psychoanalytic experience with patients, but they were also linked to his own life history (Blatt, 1974; Blatt, 2004). Specifically, Blatt recounts how, in the treatment of two patients with depression, he was struck by the very different nature of the types of conflicts his patients were struggling with. 
While the depressive experience of one of his patients seemed to primarily revolve around issues of relatedness, such as feelings of abandonment and rejection, the other patient seemed to struggle mainly with issues related to self-definition, such as achievement, guilt, and control. Blatt (2004) goes on to recall that while the depression of the second patient resembled most closely the typical description of depression in much of 20th century psychiatry, descriptions of the depressive experience of the first patient were, with some important exceptions, remarkably absent from the psychoanalytic and psychiatric literature. Yet, it reminded him of seeing his own father cry over his mother's grave, seemingly feeling desperate and abandoned. This experience, Blatt recounted, probably made him more aware of and attuned to depressive experiences related to feelings of abandonment and rejection, and thus it is likely to have influenced his theorizing about the importance of such experiences.

What perhaps distinguished Blatt most from many of his contemporary psychoanalytic thinkers and clinicians was that he decided to put his ideas and formulation to the empirical test. Together with colleagues, he developed the Depressive Experiences Questionnaire (DEQ) (Blatt et al., 1976). This 66-item self-report questionnaire was designed to capture maladaptive expressions of relatedness, as evidenced in high levels of Dependency, and maladaptive expressions of self-definition, as captured by the questionnaire's Self-Criticism scale. Several other self-report measures assessing these dimensions have emerged since (for reviews see Luyten et al., 2005; Zuroff et al., 2004), and more recently, observer-rated scales of these dimensions have also been developed that capture different levels of concerns with self-definition and relatedness at different developmental levels. These include the Differentiation-Relatedness Scale (Diamond et al., 2011), the Prototype Matching of Anaclitic and Introjective Personality Orientation (Werbart and Forsström, 2014), and the Anaclitic-Introjective Depression Assessment (Rost et al., 
2016). Similarly, within interpersonal theories, various measures tapping into different facets of relatedness and self-definition have been developed and validated (Locke, 2011), and attachment research has focused on interview-based measures, such as the Adult Attachment Interview and the Child Attachment Interview, and experimental paradigms, including the Strange Situation (Ravitz et al., 2010).

A large body of research using these measures has provided evidence supporting the main assumptions of Blatt's two-polarities model outlined above in understanding both normal and disrupted psychological development from childhood to adolescence and adulthood (for reviews see Luyten and Blatt, 2013; Luyten et al., 2005; Zuroff et al., 2004). These views rank among the best-researched and -supported psychoanalytic theories, and have also inspired much research on process-outcome relationships, as Blatt assumed that similar processes were at work in psychotherapy as in normal human development (Blatt et al., 2010).

Overall, this research has shown the particularly destructive nature of self-criticism (Blatt, 1995; Shahar, 2015), while dependency seems to be much more of a double-edged sword. Associations between self-criticism and psychopathology and psychological functioning more generally are typically stronger for self-criticism than for dependency. Studies suggest that this seems to be in large part due to the fact that highly dependent/anaclitic individuals are able to develop and maintain, although often with much ambivalence, supportive relationships with others, whereas highly introjective/self-critical individuals typically lack the capacity to turn to others - particularly in times of need. These findings again appear to confirm Blatt's intuition that human beings are fundamentally interpersonal, and that those individuals who show severe impairments in the capacity to turn to others in times of need (such as highly introjective individuals) often seem to fare 
particularly poorly in life, or at least pay a very heavy price for their inability to acknowledge their connectness to others.

Studies have confirmed that high levels of dependency and self-criticism are both associated with dysfunctional interpersonal transactional cycles (Kiesler, 1983), leading to exactly the behaviors and reactions by others that the individual fears and tends to avoid, which in turn strengthen the individual's (distorted) expectations about him/herself and others. Highly dependent/anaclitic individuals may be able to generate a positive social environment, but their claiming relational style often leads to irritation, anger, and resentment in others, which then may lead to actual rejection and abandonment, confirming these individuals' strong fear of being rejected and abandoned. Highly self-critical/introjective individuals, by contrast, tend to be ambivalent, critical, and distrustful of others, because of their fear of criticism and disapproval. Yet, this leads them to be perceived by others as cold, distant, and aloof, which confirms the individual's belief that others do not like them and disapprove of them.

Studies based on Blatt's assumptions have amply demonstrated the impact of these dysfunctional transactional cycles on the nature of the therapeutic relationship and therapeutic response (Blatt et al., 2010). Dependent/anaclitic and self-critical/introjective individuals bring very different needs and expectations into therapy, and tend to perceive the therapeutic relationship in personality-congruent ways. As demonstrated by systematic research studies, the identification, articulation, and working through of these transference reactions is crucial to establish a working alliance and for long-term change. Studies in this context, consistent with Blatt's assumptions, suggest that patients primarily preoccupied with issues of relatedness are responsive mainly to supportive dimensions in therapy. In contrast, patients primarily preoccupied with issues of self-definition are more responsive to interpretive-exploratory dimensions (Blatt et al., 2010). In this view, successful treatment, 
regardless of the type of treatment, involves the reactivation of a normal synergistic developmental process in which interpersonal experiences in the therapeutic relationship, as in normal personality development, foster feelings of autonomy and self-definition, which in turn foster interpersonal relatedness.

It is important to point out that problems with relatedness and self-definition thus are transdiagnostic vulnerability factors that are implicated in vulnerability for psychopathology across the life span (for reviews, see Blatt, 2008; Blatt and Luyten, 2010; Egan et al., 2011; Zuroff et al., 2004). These personality factors thus explain, at least in part, the notable heterogeneity within and across different disorders. For instance, in depressed patients, at least four subtypes have been distinguished based on these views: individuals with clinical depression falling within a higher-functioning, less maladaptive, anaclitic Needy Depression and introjective Self-Critical Depression prototype, versus clinically depressed patients falling into two lower-functioning, more maladaptive, anaclitic Submissive Depression and introjective Dismissive Depression prototypes (Rost et al., 2016).

Similarly, studies suggest that personality disorders can be organized into a cluster focused around issues of relatedness (an "anaclitic" configuration) and a cluster focused around issues of self-definition (an "introjective" configuration) (Blatt and Luyten, 2010). The DSM dependent, histrionic, and borderline personality disorders typically fall into the former cluster, while the antisocial, narcissistic, paranoid, schizoid, schizotypic, avoidant, and obsessive-compulsive personality disorders typically fall within the second cluster. These findings have influenced proposals for a radical change in the way personality pathology is organized and assessed in DSM 5 and subsequent editions, emphasizing the centrality of interpersonal relatedness and self-definition in understanding and classifying personality disorders (Bender et al., 2011) 
Studies based on two-polarities models have also increased our understanding of the intergenerational transmission of vulnerability for psychopathology, suggesting that disproportionate concerns with issues of relatedness and self-definition are transmitted from one generation to the next. This has been demonstrated in studies of children as young as 4 months (Beebe et al., 2007) to studies focusing on adolescents (Soenens et al., 2010). A study by Besser and Priel (2005) in a community sample even showed that excessive preoccupation with relatedness and self-definition was transmitted from mothers to their daughters and granddaughters. Further evidence for the intergenerational transmission of preoccupation with relatedness and self-definition has emerged from attachment research (Verhage et al., 2016).

\section{Two polarities of experience, biology, and health}

Consistent with Blatt's emphasis on the synergistic interaction between relatedness and selfdefinition, neurobiological research has suggested considerable overlap in the neural circuitry involved in social cognition with regard to the development of relatedness and the self (Lombardo et al., 2010).

Neural circuits involved in the capacity for relatedness primarily consist of a mesocorticolimbic dopaminergic reward circuit and hypothalamic-midbrain-limbicparalimbic-cortical circuits (Fonagy et al., 2011; Panksepp, 1998; Swain et al., 2007; Vrticka and Vuilleumier, 2012). Key neuromodulators in these circuits, including neuropeptides such as oxytocin and vasopressin, seem to be involved in the rewarding features of affiliative behaviors (ranging from parental care to pair-bonding and sexual behavior), in social cognitive capacities underpinning relationships, and in the regulation of behavioral and neuroendocrinological responses to stress (Neumann, 2008). Specifically, secure attachment experiences seem to reinforce affiliative behavior, resulting in "broaden and build" 
(Fredrickson, 2001) cycles: broadening results from the fact that secure attachment experiences encourage explorative behavior; building, in turn, results from the fact that attachment relationships are the "playground" where skills and resources are developed. Hence, in normative development, experiences of relatedness are intrinsically related to the development of feelings of autonomy, competence, and identity (Fonagy and Luyten, 2009; Fredrickson, 2001; Mikulincer and Shaver, 2007). By contrast, disrupted development is associated with a disturbance in the dialectic interaction in the development of neural circuits involved in the processing information about self and others.

These assumptions are further reinforced by findings that neural circuits involved in reflecting on the self and others are closely intertwined. These circuits include cortical midline structures such as the medial prefrontal cortex, posterior cingulate, precuneus, and temporoparietal junction (Lieberman, 2007; Lombardo et al., 2011; Lombardo et al., 2010; for a meta-analysis, see Northoff et al., 2006).

Consistent with these assumptions, there is now good evidence from research in both humans and animals that attachment experiences play a key role in programming the development of the hypothalamic-pituitary-adrenal axis, the main human stress system that is underpins our capacity to deal with conflict and adversity, and in associated biological systems such as the immune system and pain-processing systems (Lupien et al., 2009a; Luyten et al., 2013; Sbarra and Hazan, 2008). These early programming effects are currently thought to be implicated in explaining vulnerability not only to psychiatric but also to functional somatic and somatic disorders across the life span (Lupien et al., 2009b). Consistent with these views, research has shown how both highly self-critical/introjective and highly dependent/anaclitic individuals are more prone to develop a host of functional somatic problems and disorders, with most of the evidence again being for the role of self- 
critical/introjective features (Luyten et al., 2013; Shahar, 2015). Disruptions in stress regulation and associated biological systems have been shown to mediate these relationships.

\section{Conclusions}

In contrast to more static symptom- or disorder-centered approaches, Blatt's two-polarities model fundamentally proposes that psychopathology reflects attempts to achieve some stability in response to developmental disruptions by becoming preoccupied, at different developmental levels, with one or the other of the developmental dimensions of interpersonal relatedness or self-definition (Blatt, 2008; Luyten et al., 2012).

Although Blatt's views have gone through a series of reformulations over the years, the basic formulations of his two-polarities model continue to provide a productive theoretical, empirical and clinical framework in conceptualizing continuities between normal and disrupted personality development, vulnerability for psychopathology, mental and physical health, and responsiveness to psychotherapy. Above all, they also demonstrate the ability of psychoanalytic thinking to influence mainstream psychology and psychiatry because of its dedication to systematic empirical research, while at the same time being solidly rooted in psychoanalytic theory and experience. 


\section{References}

AMERICAN PSYCHIATRIC Association. (2013). Diagnostic and Statistical Manual of Mental Disorders. Arlington, VA: American Psychiatric Press.

Angyal, A. (1951). Neurosis and Treatment: A Holistic Theory. New York: Wiley.

BAKAN, D. (1966). The Duality of Human Existence. Chicago: Rand McNally.

BECK, A.T. (1983). Cognitive therapy of depression: New perspectives. In: Treatment of Depression: Old Controversies and New Approaches ed. P.J. Clayton \& J.E. Barrett. New York: Raven Press, pp. 265-290.

Beebe, B., Jaffe, J., Buck, K., Chen, H., Cohen, P., Blatt, S., Kaminer, T., Feldstein, S., \& ANDREWS, H. (2007). Six-week postpartum maternal self-criticism and dependency and 4-month mother-infant self- and interactive contingencies. Developmental Psychology 43:1360-1376.

Bender, D.S., MoRey, L.C., \& SKOdOL, A.E. (2011). Toward a model for assessing level of personality functioning in DSM-5, Part I: A review of theory and methods. Journal of Personality Assessment 93:332-346.

Besser, A., \& Priel, B. (2005). The apple does not fall far from the tree: Attachment styles and personality vulnerabilities to depression in three generations of women. Personality and Social Psychology Bulletin 31:1052-1073.

BLATT, S.J. (1974). Levels of object representation in anaclitic and introjective depression. Psychoanalytic Study of the Child 29:107-157.

BlATt, S.J. (1995). The destructiveness of perfectionism. Implications for the treatment of depression. American Psychologist 50:1003-1020.

BLATT, S.J. (2004). Experiences of Depression: Theoretical, Clinical, and Research Perspectives. Washington, DC: American Psychological Association. 
Blatt, S.J. (2008). Polarities of Experience: Relatedness and Self Definition in Personality Development, Psychopathology, and the Therapeutic Process. Washington, DC: American Psychological Association.

BLATT, S.J., \& BEHRENDS, R.S. (1987). Internalization, separation-individuation, and the nature of therapeutic action. International Journal of Psychoanalysis 68:279-297.

BLATT, S.J., D’AfFLITTI, J.P., \& QuinLAN, D.M. (1976). Experiences of depression in normal young adults. Journal of Abnormal Psychology 85:383-389.

Blatt, S.J., \& LUYTEN, P. (2009). A structural-developmental psychodynamic approach to psychopathology: Two polarities of experience across the life span. Development and Psychopathology 21:793-814.

BLATT, S.J., \& LUYTEN, P. (2010). Reactivating the psychodynamic approach to classify psychopathology. In: Contemporary Directions in Psychopathology. Scientific Foundations of the DSM-V and ICD-11 ed. T. Millon, R.F. Krueger \& E. Simonsen. New York: Guilford Press, pp. 483-514.

BLATT, S.J., \& SHICHMAN, S. (1983). Two primary configurations of psychopathology. Psychoanalysis and Contemporary Thought 6:187-254.

BLATT, S.J., \& SHICHMANN, S. (1983). Two primary configurations of psychopathology. Psychoanalysis and Contemporary Thought 6:187-254.

Blatt, S.J., ZurofF, D.C., HAWLey, L.L., \& AuerbaCh, J.S. (2010). Predictors of sustained therapeutic change. Psychotherapy Research 20:37-54.

ClARK, D.A., \& BECK, A.T. (1999). Scientific Foundations of Cognitive Theory and Therapy of Depression. New York: John Wiley \& Sons.

DECI, E.L., \& RYAN, R.M. (2012). Self-determination theory. In: Handbook of Theories of Social Psychology, ed. P.A.M.V. Lange, A.W. Kruglanski \& E.T. Higgins. Thousand Oaks, CA: Sage, pp. 416-437. 
Diamond, D., Blatt, S.J., Stayner, D.A., \& Kaslow, N. (2011). DifferentiationRelatedness of Object Representations. Unpublished Research Manual. Yale University, New Haven, CT.

EgAN, S.J., WADE, T.D., \& SHAFRAN, R. (2011). Perfectionism as a transdiagnostic process: A clinical review. Clinical Psychology Review 31:203-212.

FonAGy, P., \& LUYTEN, P. (2009). A developmental, mentalization-based approach to the understanding and treatment of borderline personality disorder. Development and Psychopathology 21:1355-1381.

FonAGy, P., LUYTEN, P., \& STRATHEARN, L. (2011). Borderline personality disorder, mentalization, and the neurobiology of attachment. Infant Mental Health Journal $32: 47-69$.

FREDRICKSON, B.L. (2001). The role of positive emotions in positive psychology. The broaden-and-build theory of positive emotions. American Psychologist 56:218-226.

Freud, A. (1963). The concept of developmental lines. Psychoanalytic Study of the Child $18: 245-265$.

FREUd, S. (1905). Three essays on the theory of sexuality. In: The Standard Edition of the Complete Psychological Works of Sigmund Freud, ed. J. Strachey. London: Hogarth Press, pp. 123-230.

FREUD, S. (1915). Instincts and their vicissitudes. In: The Standard Edition of the Complete Psychological Works of Sigmund Freud, ed. J. Strachey. London: Hogarth Press, pp. 109-140.

KAZDIN, A.E. (2011). Evidence-based treatment research: Advances, limitations, and next steps. American Psychologist 66:685-698.

KIESLER, D.J. (1983). The 1982 interpersonal circle: A taxonomy for complementarity in human transactions. Psychological Review 90:185-214. 
Kitayama, S., Markus, H.R., Matsumoto, H., \& NorASAKKUnKit, V. (1997). Individual and collective processes in the construction of the self: Self-enhancement in the United States and self-criticism in Japan. Journal of Personality and Social Psychology 72:1245-1267.

Lakatos, I. (1976). Proofs and Refutations: The Logic of Mathematical Discovery. Cambridge: Cambridge University Press.

LIEBERMAN, M.D. (2007). Social cognitive neuroscience: A review of core processes. Annual Review of Psychology 58:259-289.

LOCKE, D. (2011). Circumplex measures of interpersonal constructs. In: Handbook of Interpersonal Psychology: Theory, Research, Assessment, and Therapeutic Intervention, ed. L.M. Horowitz \& S. Strack. Hoboken, NJ: John Wiley \& Sons, pp. 313-324.

Lombardo, M.V., Chakrabarti, B., Bullmore, E.T., \& Baron-Cohen, S. (2011). Specialization of right temporo-parietal junction for mentalizing and its relation to social impairments in autism. Neuroimage 56:1832-1838.

Lombardo, M.V., Chakrabarti, B., Bullmore, E.T., Wheelwright, S.J., SAdeK, S.A., SUCKLING, J., \& BARON-COHEN, S. (2010). Shared neural circuits for mentalizing about the self and others. Journal of Cognitive Neuroscience 22:1623-1635.

LuPIEn, S.J., McEwen, B.S., GunNAR, M.R., \& HeIM, C. (2009a). Effects of stress throughout the lifespan on the brain, behaviour and cognition. Nature Reviews Neuroscience 10:434-445.

LuPIEN, S.J., McEwEN, B.S., GunNAR, M.R., \& HeIM, C. (2009b). Effects of stress throughout the lifespan on the brain, behaviour and cognition. Nature Reviews Neuroscience 10:434-445. 
LuYTEN, P., \& Blatt, S.J. (2007). Looking back towards the future: Is it time to change the DSM approach to psychiatric disorders? The case of depression. Psychiatry: Interpersonal and Biological Processes 70:85-99.

LUYTEN, P., \& BLATT, S.J. (2011). Integrating theory-driven and empirically-derived models of personality development and psychopathology: A proposal for DSM-V. Clinical Psychology Review 31:52-68.

LUYTEN, P., \& BLATT, S.J. (2013). Interpersonal relatedness and self-definition in normal and disrupted personality development: Retrospect and prospect. American Psychologist 68:172-183.

LUYTEN, P., \& BLATT, S.J. (2015). A hierarchical multiple-level approach to the assessment of interpersonal relatedness and self-definition: Implications for research, clinical practice, and DSM planning. Journal of Personality Assessment:1-9.

Luyten, P., Blatt, S.J., \& MAYES, L.C. (2008). Process and outcome in psychoanalytic psychotherapy research: The need for a (relatively) new paradigm. In: Handbook of Evidence-Based Psychodynamic Psychotherapy. Bridging the Gap between Science and Practice, ed. R.A. Levy, J.S. Ablon \& H. Kächele. 2nd ed. New York: Humana Press/Springer, pp.

LuYTen, P., CoRveleyn, J., \& BlatT, S.J. (2005). The convergence among psychodynamic and cognitive-behavioral theories of depression: A critical overview of empirical research. In: The Theory and Treatment of Depression: Towards a Dynamic Interactionism Model, ed. J. Corveleyn, P. Luyten \& S.J. Blatt. Mahwah, NJ: Lawrence Erlbaum Associates, pp. 107-147.

LuYten, P., MAYes, L.C., TARget, M., \& Fonagy, P. (2012). Developmental research. In: Textbook of Psychoanalysis, ed. G.O. Gabbard, B. Litowitz \& P. Williams. 2nd ed. Washington, DC: American Psychiatric Press, pp. 423-442. 
Luyten, P., Van Houdenhove, B., Lemma, A., TArget, M., \& Fonagy, P. (2013).

Vulnerability for functional somatic disorders: A contemporary psychodynamic approach. Journal of Psychotherapy Integration 23:14-27.

MARKUS, H.R., \& KITAYAMA, S. (1991). Culture and the self: Implications for cognition, emotion and motivation. Psychological Review 98:224-253.

McAdAms, D.P. (1985). Power, Intimacy, and the Life Story: Personological Inquiries into Identity. Homewood, IL: Dorsey.

MCClelland, D.C. (1985). Human Motivation. Cambridge: Cambridge University Press.

MCHugh, R.K., MurRay, H.W., \& BARLOW, D.H. (2009). Balancing fidelity and adaptation in the dissemination of empirically-supported treatments: The promise of transdiagnostic interventions. Behaviour Research and Therapy 47:946-953.

McWilliams, N. (2011). Psychoanalytic Diagnosis: Understanding Personality Structure in the Clinical Process. London/New York: Guilford Press.

Mikulincer, M., \& SHAVer, P.R. (2007). Attachment in Adulthood: Structure, Dynamics, and Change. New York: Guilford Publications.

NEUMANN, I.D. (2008). Brain oxytocin: A key regulator of emotional and social behaviours in both females and males. Journal of Neuroendocrinology 20:858-865.

Northoff, G., Heinzel, A., DE Greck, M., Bermpohl, F., Dobrowolny, H., \& PAnKsepp, J. (2006). Self-referential processing in our brain — a meta-analysis of imaging studies on the self. Neuroimage 31:440-457.

PANKSEPP, J. (1998). Affective Neuroscience: The Foundations of Human and Animal Emotions. Oxford: Oxford University Press.

PINCUS, A.L. (2005). A contemporary integrative interpersonal theory of personality disorders. In: Major Theories of Personality Disorder, ed. M.F. Lenzenweger \& J.F. Clarkin. 2nd ed. New York: Guilford Press, pp. 282-331. 
Ravitz, P., Maunder, R., Hunter, J., Sthankiya, B., \& LAncee, W. (2010). Adult attachment measures: A 25-year review. Journal of Psychosomatic Research 69:419432.

Rost, F., Fonagy, P., \& Luyten, P. (2016). The Anaclitic-Introjective Depression Assessment: Development and Preliminary Validity of an Observer-Rated Measure. Manuscript submitted for publication.

RYAN, R.M., \& DECI, E.L. (2000). Self-determination theory and the facilitation of intrinsic motivation, social development, and well-being. American Psychologist 55:68-78.

SAFRAn, J.D., Muran, J.C., \& EUbANKS-CARTER, C. (2011). Repairing alliance ruptures. Psychotherapy 48:80-87.

Safran, J.D., Muran, J.C., SAmstag, L.W., \& SteVen, C. (2002). Repairing alliance ruptures. In: Psychotherapy Relationships that Work, ed. J.C. Norcross. Oxford: Oxford University Press, pp. 235-254.

SBARRA, D.A., \& HAZAN, C. (2008). Coregulation, dysregulation, self-regulation: An integrative analysis and empirical agenda for understanding adult attachment, separation, loss, and recovery. Personality and Social Psychology Review 12:141-167.

SHAHAR, G. (2015). Erosion: The Psychopathology of Self-Criticism. Oxford: Oxford University Press.

SIBLEY, C. (2007). The association between working models of attachment and personality: Toward an integrative framework operationalizing global relational models. Journal of Research in Personality 41:90-109.

SKODOL, A.E., \& BENDER, D.S. (2009). The future of personality disorders in DSM-V? American Journal of Psychiatry 166:388-391.

Soenens, B., VAnsteenkiste, M., \& Luyten, P. (2010). Towards a domain-specific approach to the study of parental psychological control: Distinguishing between 
dependency-oriented and achievement-oriented psychological control. Journal of Personality 78:217-256.

SPITZ, R.A. (1945). Hospitalism: An inquiry into the genesis of psychiatric conditions in early childhood. Psychoanalytic Study of the Child 1:53-74.

Swain, J.E., Lorberbaum, J.P., Kose, S., \& Strathearn, L. (2007). Brain basis of early parent-infant interactions: Psychology, physiology, and in vivo functional neuroimaging studies. Journal of Child Psychology and Psychiatry 48:262-287.

TRIANDIS, H.C. (2001). Individualism-collectivism and personality. Journal of Personality 69:907-924.

Verhage, M.L., Schuengel, C., Madigan, S., Fearon, R.M., Oosterman, M., Cassibba, R., BAKERMANS-KRANENBURG, M.J., \& VAN, I.M.H. (2016). Narrowing the transmission gap: A synthesis of three decades of research on intergenerational transmission of attachment. Psychological Bulletin 142:337-366.

VRTickA, P., \& Vuilleumier, P. (2012). Neuroscience of human social interactions and adult attachment style. Frontiers in Human Neuroscience 6:212.

WERBART, A., \& FORSSTRÖM, D. (2014). Changes in anaclitic-introjective personality dimensions, outcomes and psychoanalytic technique: A multi-case study. Psychoanalytic Psychotherapy 28:397-410.

Zuroff, D.C., Mongrain, M., \& SANTOR, D.A. (2004). Conceptualizing and measuring personality vulnerability to depression: Commentary on Coyne and Whiffen (1995). Psychological Bulletin 130:453-472. 